\title{
Periodic Orbits for a Three-Dimensional Biological Differential Systems
}

\author{
Renato Colucci ${ }^{1}$ and Daniel Nuñez ${ }^{2}$ \\ ${ }^{1}$ Departamento de Matemáticas, Pontificia Universidad Javeriana, Carrera 7, No. 43-82, Bogotá, Colombia \\ ${ }^{2}$ Departamento de Ciencias Naturales y Matemáticas, Facultad de Ingeniería, Pontificia Universidad Javeriana, Cali, Colombia
}

Correspondence should be addressed to Renato Colucci; renatocolucci@hotmail.com

Received 3 June 2013; Accepted 7 June 2013

Academic Editor: Luca Guerrini

Copyright @ 2013 R. Colucci and D. Nuñez. This is an open access article distributed under the Creative Commons Attribution License, which permits unrestricted use, distribution, and reproduction in any medium, provided the original work is properly cited.

We study the existence of periodic orbit for a differential system describing the effects of indirect predation over two preys. Besides discussing a generalized version of the model, we present some remarks and numerical experiments for the nonautonomous version of the two models.

\section{Introduction}

The role of indirect effects in biology has been extensively studied in the last decades (see [1-7]); for example, in the case of predation (see [8]), the predator can alter the morphology (see [9]) or the behavior of the preys. The preys, in order to reduce the possibility of contacts with the predators, could modify their normal conduct by reducing their activity or by hiding themselves for long time. There are many types of indirect effects (see [3] for a detailed discussion). Another interesting case is the refuge indirect effect (see [10] for an example); anyway it is of great interest trying to describe the indirect interactions in population dynamics. In [11] a model was proposed including indirect effects, modeling the effects of predator Daphnia over two groups of Phytoplankton of different morphology (see $[12,13])$, having Phosphorous as resource (see [13] or [7]). The system takes the following form

$$
\begin{aligned}
Z^{\prime} & =Z\left(-e+u_{c} C+u_{g} G\right), \\
C^{\prime} & =C\left(a_{c} P-u_{c} Z\right)-m C Z, \\
G^{\prime} & =G\left(a_{g} P-u_{g} Z\right)+m C Z, \\
P^{\prime} & =P\left(-a_{g} G-a_{c} C\right)+e Z,
\end{aligned}
$$

where $u_{g}, u_{c}, a_{c}, a_{g}, e, m \in \mathbb{R}$ are positive parameters and where $(Z, C, G, P) \subset \mathbb{R}_{+}^{4}$.
In the previous system $Z$ represents the density of population of a predator (Daphnia or Zooplankton) that predates the preys (Phytoplankton) $C$ and $G$ that are of different size, in particular $C$ being of a smaller size than $G$. The variable $P$ represents the amount of resources (Phosphorous) for the preys $C$ and $G$. The system admits a constant of motion $I(t)=$ $Z+C+G+P$, and then we can reduce the degree of freedom of the problem by fixing a value of the first integral, $I(t)=I_{0}$. The system can be rewritten in the following way:

$$
\begin{gathered}
Z^{\prime}=Z\left(-e+u_{c} C+u_{g} G\right), \\
C^{\prime}=C\left[a_{c} I_{0}-\left(a_{c}+u_{c}\right) Z-a_{c} C-a_{c} G\right]-m C Z, \\
G^{\prime}=G\left[a_{g} I_{0}-\left(a_{g}+u_{g}\right) Z-a_{g} C-a_{g} G\right]+m C Z .
\end{gathered}
$$

The dynamics of system (2) have been studied in [14] by using Persistence Theory (see $[15,16])$ and in particular an acyclicity approach. It has been shown that in absence (i.e., $m=0$ ) of the terms that describe indirect effects, the system (2) does not admits coexistence of the three populations, while for $m>$ 0 and for an appropriate choice of the parameters, the system admits coexistence. The paper [14] suggests the importance of indirect effects in describing cases of coexistence with the 
parameter $m$ being a bifurcation parameter for coexistence. In particular under the hypotheses

$$
\begin{gathered}
I_{0}>\max \left\{\frac{e}{u_{c}}, \frac{e}{u_{g}}\right\}, \\
M=a_{c} u_{g}-a_{g} u_{c}-a_{g} m>0,
\end{gathered}
$$

the system is uniformly persistent. Under the previous hypotheses the system admits an attractor $\mathscr{A}$ that lies at positive distance from $\partial \mathbb{R}_{+}^{3}$ and inside the set

$$
\Delta=\left\{(Z, C, G) \in \mathbb{R}_{+}^{3}: Z+C+G \leq I_{0}\right\} .
$$

In particular the boundary fixed point

$$
\begin{gathered}
P_{0}=(0,0,0), \quad P_{2}=\left(0, I_{0}, 0\right), \quad P_{3}=\left(0,0, I_{0}\right), \\
P_{4}=\left(\frac{a_{g}}{a_{g}+u_{g}}\left[I_{0}-\frac{e}{u_{g}}\right], 0, \frac{e}{u_{g}}\right)
\end{gathered}
$$

and the segment of fixed point

$$
S:=\left\{(Z, C, G) \in \mathbb{R}^{3}: Z=0, C+G=I_{0}\right\}
$$

are all instable and their stable and central manifolds are all on $\partial R_{+}^{3}$. Under the hypotheses (3)-(4) the set $\Delta$ always contains an interior fixed point $Q=\left(Z_{Q}, C_{Q}, G_{Q}\right)$ as follows:

$$
\begin{gathered}
G_{\mathrm{Q}}=\left\{\frac{e / u_{c}}{M / a_{c} m+u_{g} / u_{c}}\right\}, \\
C_{\mathrm{Q}}=\frac{M}{a_{c} m}\left\{\frac{e / u_{c}}{M / a_{c} m+u_{g} / u_{c}}\right\}, \\
Z_{\mathrm{Q}}=\frac{a_{c}}{m_{c}}\left\{I_{0}-\left(\frac{M}{m a_{c}}+1\right)\left(\frac{e / u_{c}}{M / a_{c} m+u_{g} / u_{c}}\right)\right\},
\end{gathered}
$$

whose stability character has not been investigated in [14]. The matrix $A(Q)$ of the linearized system at $Q$ is

$$
\left(\begin{array}{ccc}
0 & u_{c} Z_{Q} & u_{g} Z_{Q} \\
-\frac{m_{c} M}{m a_{c}} D & -\frac{M}{m} D & -\frac{M}{m} D \\
-\frac{a_{g} m_{c}}{a_{c}} D & \frac{m}{m_{c}} a_{c}\left\{I_{0}-\frac{m_{g}}{m} D\right\} & -\frac{M}{a_{c}} Z_{\mathrm{Q}}-a_{g} D
\end{array}\right)
$$

where $m_{c}=m+a_{c}+u_{c}$ and $m_{g}=m+a_{g}+u_{g}$ and $D=G_{Q}$. By numerical experiments (see [14]) it was pointed out that the attractor $\mathscr{A}$ should take the following forms:

(1) the interior fixed point $Q$;

(2) a periodic orbit;

(3) a bidimensional (possibly chaotic) set.

In this work we are concerned with the proof of existence of periodic orbits (see $[17,18]$ for examples in the classical case), and we will use Hopf bifurcation theory in order to prove existence of periodic orbits. Since the system depends on 7 parameters, the conditions to have Hopf bifurcation are quite complicated and for sake of simplicity we also present a particular case in which all the parameters are fixed but $I_{0}$. In general cases we can use the following remark to facilitate our analysis.

Remark 1. The trace of the matrix $A(Q)$ satisfies the following inequality

$$
\operatorname{Tr}(A(Q))=-\frac{M}{a_{c}} Z_{Q}-G_{Q}\left(a_{g}+\frac{M}{m}\right)<0 ;
$$

then at least one eigenvalue has negative real part and as a consequence fold-Hopf bifurcation is not possible.

The rest of the paper is organized as follows. In Section 2 we present general condition to have Hopf bifurcation, while in Section 3 we give an example fixing all the parameters except for $I_{0}$. In Section 4 we consider a nonautonomous version of the model and present several numerical experiments in order to study the structure of the attractor. In the last case we discuss a generalized version of the model, both autonomous and nonautonomous, and present some numerical experiments.

\section{Condition for Hopf Bifurcation}

We consider the characteristic polynomial of the matrix $A(Q)$ as follows:

$$
x^{3}+\alpha x^{2}+\beta x+\gamma=0
$$

where

$$
\begin{aligned}
& \alpha=- \operatorname{Tr}[A(Q)] \\
&=\frac{M}{m_{c}}\left\{I_{0}-D\left[\left(\frac{M}{a_{c} m}+1\right)-\frac{m_{c} a_{g}}{M}-\frac{m_{c}}{m}\right]\right\}, \\
& \beta= Z(A(Q)) \\
&= \frac{M D}{m}\left\{I_{0}-\left(\frac{M}{a_{c} m}+1\right) D\right\} \\
& \times\left\{u_{c}+\frac{M}{m_{c}}+\frac{u_{g} a_{g} m}{M}+\frac{m a_{c}}{m_{c}}\right\}, \\
& \gamma=-\operatorname{Det}[A(Q)]=\frac{e M a_{c}}{m_{c}}\left\{I_{0}-\left(\frac{M}{a_{c} m}+1\right) D\right\}^{2} .
\end{aligned}
$$

A necessary condition for Hopf bifurcation is that each of the previous coefficients has to be positive. It easy to verify that $\alpha, \beta, \gamma>0$ if

$$
I_{0}>\left(\frac{M}{a_{c} m}+1\right) D,
$$

and the last inequality follows from hypothesis (3).

From Hurwitz-Routh criterion we need to study the sign of the term

$$
\alpha \beta-\gamma=\frac{M}{m_{c}} B\left\{I_{0}-\left(\frac{M}{a_{c} m}+1\right) D\right\} .
$$


The sign of the previous term depends only on the factor $B$; in details we have

$$
\begin{aligned}
B & =\left\{I_{0}-D\left[A-\frac{m_{c} a_{g}}{M}-\frac{m_{c}}{m}\right]\right\} F-e a_{c}\left\{I_{0}-A D\right\} \\
& =\left(F-e a_{c}\right)\left(I_{0}-I_{0}^{*}\right),
\end{aligned}
$$

where

$$
\begin{gathered}
A=\left(\frac{M}{a_{c} m}+1\right), \\
F=\frac{M D}{m}\left\{u_{c}+\frac{M}{m_{c}}+\frac{u_{g} a_{g} m}{M}+\frac{m a_{c}}{m_{c}}\right\}, \\
I_{0}^{*}=A D-D \frac{F\left(m_{c} a_{g} / M+m_{c} / m\right)}{F-e a_{c}} .
\end{gathered}
$$

From the hypothesis (3) we already have $I_{0}>A D$; then if

$$
F-e a_{c}>0
$$

we have that $\alpha \beta-\gamma>0$ for any $I_{0}>A D$, and this implies that the real parts of the eigenvalues are all negative and so the fixed point $Q$ is asymptotically stable. Moreover, all the persistence solutions converge to the fixed point $Q$.

On the contrary if

$$
F-e a_{c}<0
$$

we have that $\alpha \beta-\gamma>0$ for any $I_{0} \in\left(1, I_{0}^{*}\right)$. In this case the eigenvalues have all negative real parts and the fixed point $Q$ is asymptotically stable. If $I_{0}>I_{0}^{*}$, then $\alpha \beta-\gamma$ becomes negative and the fixed point $Q$ loses stability. Moreover a periodic orbit appears thanks to Hopf bifurcation theorem (with $I_{0}^{*}$ as the bifurcation value). Recalling that all the boundary fixed points are instable and repulsive (see [14]), we can conclude that the attractor consists of the periodic orbit generated by Hopf bifurcation. Since the system is uniformly persistent under hypotheses (3)-(4), we expect that the periodic orbit is persistent. We summarize as follows.

Proposition 2. Suppose that (3) and (4) hold. Then the matrix $A(Q)$ has one negative and two purely imaginary eigenvalues if and only if $I_{0}=I_{0}^{*}$ and condition (18) holds.

Thanks to Hopf bifurcation theorem, a periodic orbit appears. In order to illustrate this result, in the next section we will consider an example of existence of limit cycle.

\section{An Example of Existence of a Limit Cycle}

In this section we present an example of the results of Section 2 . We prove the existence of a limit cycle by considering $I_{0}$ as a bifurcation parameter and fixing the values of the others parameters.

We set

$$
u_{c}=u_{g}=e=a_{g}=m=1, \quad a_{c}=3 .
$$

We will choose $I_{0}$ in order to satisfy hypothesis (3) while hypothesis (4) is already satisfied as

$$
I_{0}>1, \quad M=1>0 .
$$

The fixed point $Q$ has the following coordinates:

$$
\left(Z_{\mathrm{Q}}, C_{\mathrm{Q}}, G_{\mathrm{Q}}\right)=\left\{\frac{3}{5}\left(I_{0}-1\right), \frac{1}{4}, \frac{3}{4}\right\},
$$

and it then exists for $I_{0}>1$ which is verified by hypothesis (3). The Jacobian matrix at point $Q$ is the following:

$$
A(Q)=\left(\begin{array}{ccc}
0 & \frac{3}{5}\left(I_{0}-1\right) & \frac{3}{5}\left(I_{0}-1\right) \\
-\frac{5}{4} & -\frac{3}{4} & -\frac{3}{4} \\
-\frac{5}{4} & \frac{3}{5}\left(I_{0}-\frac{9}{4}\right) & -\frac{1}{5}\left(I_{0}+\frac{11}{4}\right)
\end{array}\right) .
$$

The characteristic polynomial of the matrix $A(Q)$ takes the form

$$
\lambda^{3}+\alpha \lambda^{2}+\beta \lambda+\gamma=0
$$

where

$$
\begin{gathered}
\alpha=\frac{1}{5}\left\{I_{0}+\frac{13}{2}\right\}>0, \\
\beta=\frac{21}{10}\left\{I_{0}-1\right\}>0, \\
\gamma=\frac{3}{5}\left\{I_{0}-1\right\}^{2}>0,
\end{gathered}
$$

and where the three inequalities follow from hypothesis (3). We use Hurwitz-Routh criterion to study the sign of the real parts of the eigenvalues. In particular we are interested in the sign of the term

$$
\alpha \beta-\gamma=-\frac{9}{50}\left\{I_{0}-1\right\}\left\{I_{0}-\frac{37}{2}\right\},
$$

which is positive for $I_{0} \in(1,37 / 2)$ and negative for $I_{0}>37 / 2$. Then Hopf bifurcation occurs at

$$
I_{0}^{H}=\frac{37}{2}
$$

and this gives the existence of a family of periodic orbits.

In particular if $I_{0}$ satisfies hypothesis (3) and $I_{0}<I_{0}^{H}$, we have three eigenvalues with negative real part and then the fixed point is stable and $\mathscr{A}=Q$ (see Figure 1 ). In the case in which $I_{0}>I_{0}^{H}$ we have one eigenvalue with negative real part and two eigenvalues with positive real part. In this case the attractor is a periodic orbit (see Figure 2). For $I_{0}=I_{0}^{H}$ the eigenvalues are

$$
\lambda_{1}=-5, \quad \lambda_{2,3}= \pm i \frac{7 \sqrt{3}}{2}
$$

The period of the limit cycle is $T=2 \pi / \omega$, where $\omega=7 \sqrt{3} / 2$ as represented in Figure 3. 


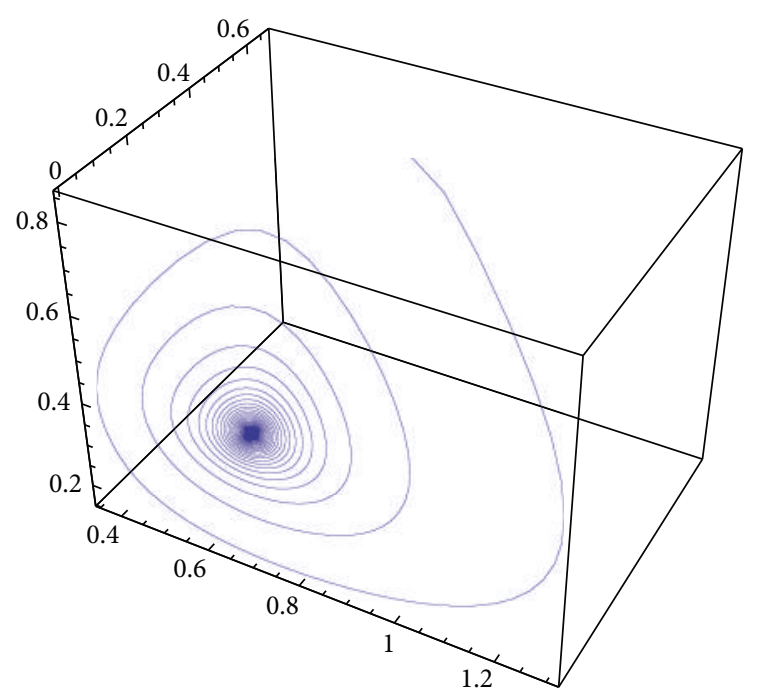

FIgURE 1: The solution of the system with $I_{0}<I_{0}^{H}$. The attractor is the fixed point $Q$.

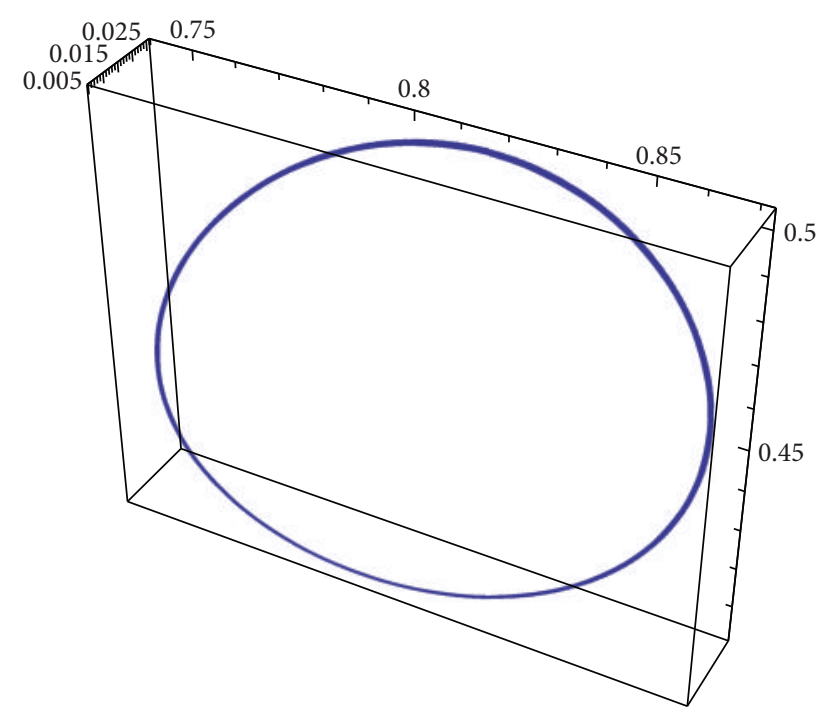

FIgURE 2: The solution of the system with $I_{0}>I_{0}^{H}$. The attractor is a periodic orbit.

We derive the equation of the central manifold in order to investigate the character of the bifurcation. We rewrite the systems with parameters as in (19) as follows:

$$
\begin{gathered}
Z^{\prime}=Z(-1+C+G), \\
C^{\prime}=C\left(3 I_{0}-5 Z-3 C-3 G\right), \\
G^{\prime}=G\left(I_{0}-2 Z-C-G\right)+C Z .
\end{gathered}
$$

We consider the following change of coordinates:

$$
\begin{gathered}
Z=x_{1}+\frac{3}{5}\left(I_{0}^{H}-1\right), \\
C=x_{2}+\frac{1}{4}, \\
G=x_{3}+\frac{3}{4},
\end{gathered}
$$

and we rewrite the system

$$
\begin{gathered}
x_{1}^{\prime}=\left(x_{1}+\frac{21}{2}\right)\left(x_{2}+x_{3}\right), \\
x_{2}^{\prime}=-\left(x_{2}+\frac{1}{4}\right)\left(5 x_{1}+3 x_{2}+3 x_{3}\right), \\
x_{3}^{\prime}=\left(x_{3}+\frac{3}{4}\right)\left(-\frac{7}{2}-2 x_{1}-x_{2}-x_{3}\right) \\
+\left(x_{2}+\frac{1}{4}\right)\left(x_{1}+\frac{21}{2}\right) .
\end{gathered}
$$

in matrix form

$$
\begin{aligned}
\left(\begin{array}{l}
x_{1}^{\prime} \\
x_{2}^{\prime} \\
x_{3}^{\prime}
\end{array}\right)= & A(Q)\left(\begin{array}{l}
x_{1} \\
x_{2} \\
x_{3}
\end{array}\right) \\
& +\left(\begin{array}{c}
x_{1}\left(x_{2}+x_{3}\right) \\
-x_{2}\left(5 x_{1}+3 x_{2}+3 x_{3}\right) \\
\left(x_{1} x_{2}-2 x_{1} x_{3}-x_{2} x_{3}-x_{3}^{2}\right)
\end{array}\right),
\end{aligned}
$$

where the matrix $A(Q)$ is calculated at $I_{0}=I_{0}^{H}$. Let $v_{1}$, $v_{2}, v_{3}$ be the eigenvectors of $A(Q)$. Consider the following transformations:

$$
\begin{gathered}
v_{1}^{\prime}=v_{1}=\left(-\frac{84}{55},-\frac{3}{11}, 1\right), \\
v_{2}^{\prime}=\frac{v_{2}+v_{3}}{2}=\left(\frac{1}{2}, \frac{1}{2}, 1\right), \\
v_{3}^{\prime}=\frac{v_{2}-v_{3}}{2 i}=\left(-\frac{3}{2} \sqrt{3}, \frac{1}{2 \sqrt{3}}, 0\right)
\end{gathered}
$$

and the change of variable

$$
\left(\begin{array}{l}
X_{1} \\
X_{2} \\
X_{3}
\end{array}\right)=P\left(\begin{array}{l}
x_{1} \\
x_{2} \\
x_{3}
\end{array}\right),
$$

where $P$ is the matrix with vectors $v_{1}^{\prime}, v_{2}^{\prime}, v_{3}^{\prime}$ as columns. Then the system becomes

$$
\begin{aligned}
\left(\begin{array}{c}
\dot{X}_{1} \\
\dot{X}_{2} \\
\dot{X}_{3}
\end{array}\right)= & P^{-1} A(Q) P\left(\begin{array}{c}
X_{1} \\
X_{2} \\
X_{3}
\end{array}\right) \\
& +P^{-1}\left(\begin{array}{c}
x_{1}\left(x_{2}+x_{3}\right) \\
-x_{2}\left(5 x_{1}+3 x_{2}+3 x_{3}\right) \\
\left(x_{1} x_{2}-2 x_{1} x_{3}-x_{2} x_{3}-x_{3}^{2}\right)
\end{array}\right) ;
\end{aligned}
$$




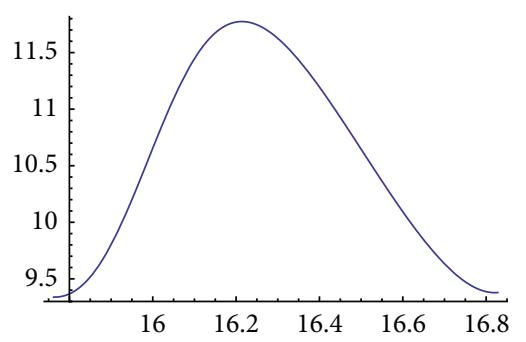

(a)

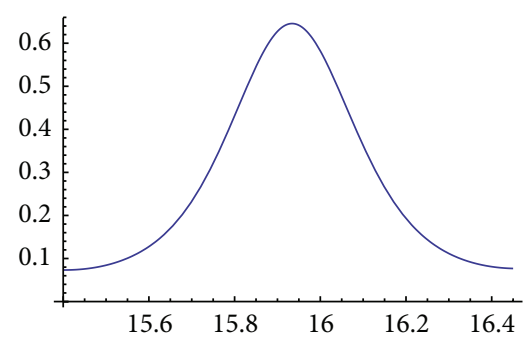

(b)

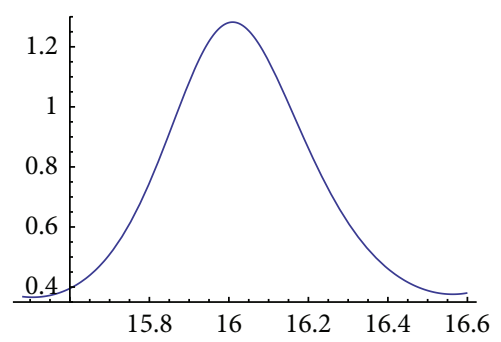

(c)

FIGURE 3: The period of the limit cycle for $I_{0}=I_{0}^{H}, T=4 \pi / 7 \sqrt{3} \simeq 1.03$.

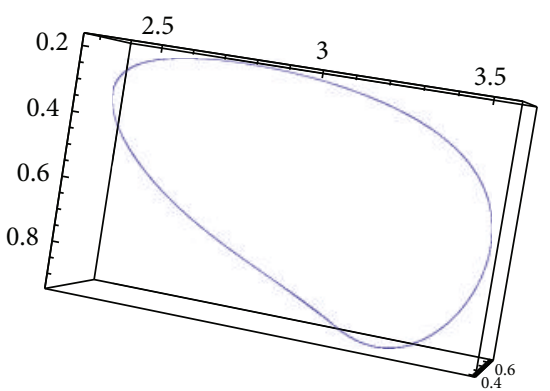

(a)

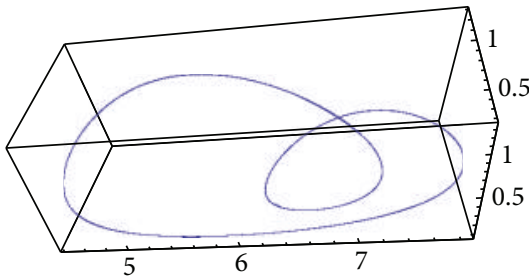

(b)

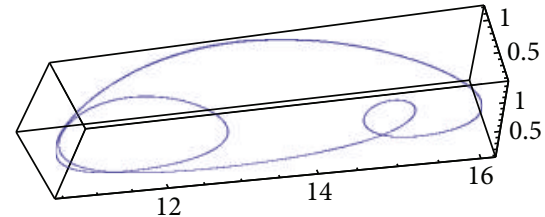

(c)

Figure 4: The solutions for $I_{0}=7,14,28$, respectively.

that is

$$
\left(\begin{array}{c}
\dot{X}_{1} \\
\dot{X}_{2} \\
\dot{X}_{3}
\end{array}\right)=\left(\begin{array}{ccc}
-5 & 0 & 0 \\
0 & 0 & \frac{7 \sqrt{3}}{2} \\
0 & -\frac{7 \sqrt{3}}{2} & 0
\end{array}\right)\left(\begin{array}{l}
X_{1} \\
X_{2} \\
X_{3}
\end{array}\right)+\left(\begin{array}{l}
f_{1} \\
f_{2} \\
f_{3}
\end{array}\right),
$$

where

$$
\begin{aligned}
f_{1}= & \frac{55\left(X_{1}+9 X_{2}-5 X_{3}\right)}{244036} \\
& \times\left\{\left((-55+85 \sqrt{3}) X_{1}-(495+223 \sqrt{3}) X_{2}\right.\right. \\
& \left.\left.+3(-73+23 \sqrt{3}) X_{3}\right)\right\}, \\
f_{2}= & \frac{55 X_{1}+495 X_{2}+219 X_{3}}{244036} \\
& \quad \times\left\{5(22+51 \sqrt{3}) X_{1}+(990-669 \sqrt{3}) X_{2}\right. \\
& \left.\quad+(-2032+207 \sqrt{3}) X_{3}\right\}, \\
f_{3}= & \frac{1}{244036}\left\{-25(988+187 \sqrt{3}) X_{1}^{2}\right. \\
& \quad+3(-131404+36795 \sqrt{3}) X_{2}^{2}
\end{aligned}
$$

$$
\begin{aligned}
& +2(60021-102821 \sqrt{3}) X_{2} X_{3} \\
& +3(15314+17687 \sqrt{3}) X_{3}^{2} \\
& -10 X_{1}\left((-5928+2981 \sqrt{3}) X_{2}\right. \\
& \left.\left.+(3211-6157 \sqrt{3}) X_{3}\right)\right\} .
\end{aligned}
$$

We consider a 2-dimensional center manifold of the type

$$
X_{1}=h\left(X_{2}, X_{3}\right)=a X_{2}^{2}+b X_{2} X_{3}+c X_{3}^{2} .
$$

Using the first equation of the system, we get

$$
\begin{aligned}
\dot{X}_{1}= & -5 X_{2}^{2}\left(a+\frac{99(495+223 \sqrt{3})}{244036}\right) \\
& -5 X_{3}^{2}\left(c+\frac{165(-73+23 \sqrt{3})}{244036}\right) \\
& +5 X_{2} X_{3}\left(-b+\frac{11(1851 \sqrt{3}+504)}{244036}\right),
\end{aligned}
$$

while using (37) and the equations of the systems, we get

$$
\dot{X}_{1}=7 \sqrt{3}\left(-\frac{b}{2} X_{2}^{2}+\frac{b}{2} X_{3}^{2}+(a-c) X_{2} X_{3}\right) \text {. }
$$




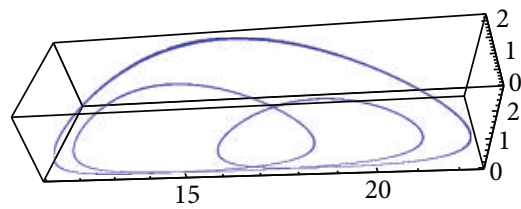

(a)

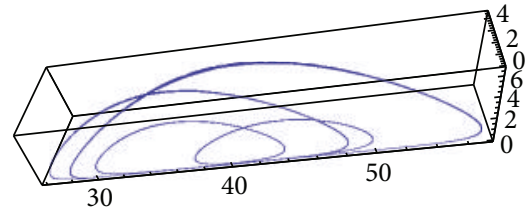

(b)

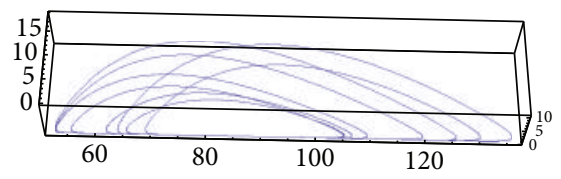

(c)

FIGURE 5: The solution for $I_{0}=35,82,177$, respectively.

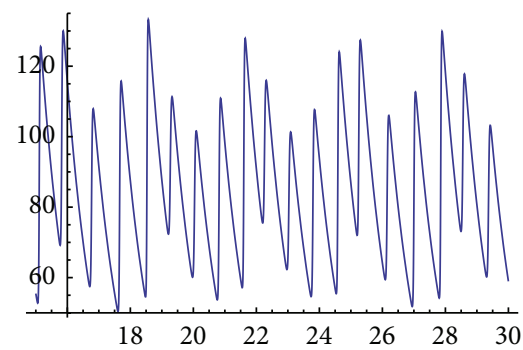

(a)

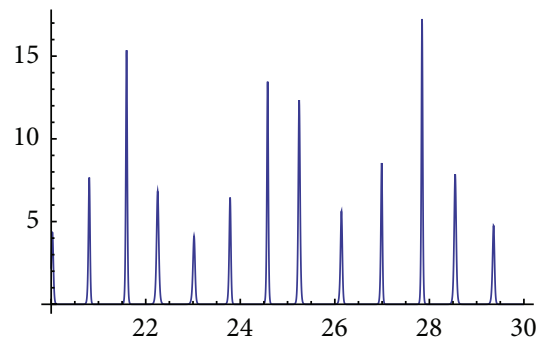

(b)

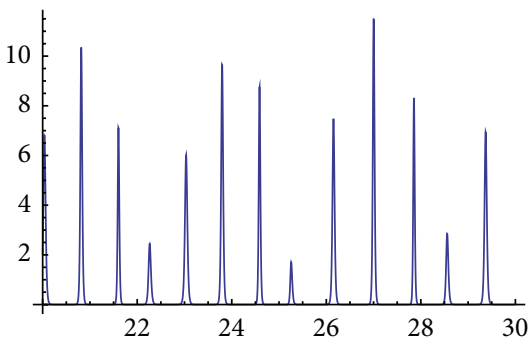

(c)

Figure 6: The solutions $Z, C, G$ as function of time $I_{0}=177$.

Equaling the coefficients, we get

$$
\begin{gathered}
-5\left(a+\frac{99(495+223 \sqrt{3})}{244036}\right)=-7 \sqrt{3} \frac{b}{2}, \\
-5\left(c+\frac{165(-73+23 \sqrt{3})}{244036}\right)=7 \sqrt{3} \frac{b}{2}, \\
5\left(-b+\frac{11(1851 \sqrt{3}+504)}{244036}\right)=7 \sqrt{3}(a-c),
\end{gathered}
$$

with solutions

$$
\begin{aligned}
& a=-\frac{99(58035+47606 \sqrt{3})}{83948384}, \\
& b=\frac{495 \sqrt{3}(5345+1386 \sqrt{3})}{41974192}, \\
& c=-\frac{33(211175+126878 \sqrt{3})}{83948384} .
\end{aligned}
$$

The system restricted on the central manifold takes the form

$$
\begin{aligned}
\left(\begin{array}{c}
\dot{X}_{2} \\
\dot{X}_{3}
\end{array}\right)= & \left(\begin{array}{cc}
0 & \frac{2133}{160} \sqrt{3815} \\
-\frac{2133}{160} \sqrt{3815} & 0
\end{array}\right)\left(\begin{array}{l}
X_{2} \\
X_{3}
\end{array}\right) \\
& +\left(\begin{array}{l}
g_{2}\left(X_{2}, X_{3}\right) \\
g_{3}\left(X_{2}, X_{3}\right)
\end{array}\right),
\end{aligned}
$$

where $g_{2}\left(X_{2}, X_{3}\right)=f_{2}\left(h\left(X_{2}, X_{3}\right), X_{2}, X_{3}\right), g_{3}\left(X_{2}, X_{3}\right)=$ $f_{3}\left(h\left(X_{2}, X_{3}\right), X_{2}, X_{3}\right)$. As remarked in the previous section the periodic orbit should persist and should be stable for $I_{0} \geq I_{0}^{H}$.

The first Lyapunov coefficient [19, page 99] can be calculated as

$$
\begin{aligned}
l_{1}(0) & =\frac{-32340518937237+21544969548257 \sqrt{3}}{501917482026688} \\
& \simeq-0.0099149,
\end{aligned}
$$

and this confirm that the Hopf bifurcation is supercritical.

\section{The Nonautonomous Case}

Since indirect effects can be of seasonal type (see [14]), it is interesting to study the case in which the constant $m$ is replaced by the time-dependent term:

$$
m|\sin t| .
$$

We consider values of the parameters for which a periodic orbit exists and make the substitution (44) in the indirect effects terms of the system

$$
\begin{gathered}
Z^{\prime}=Z\left(-e+u_{c} C+u_{g} G\right), \\
C^{\prime}=C\left[a_{c} I_{0}-\left(a_{c}+u_{c}\right) Z-a_{c} C-a_{c} G\right]-m|\sin t| C Z, \\
G^{\prime}=G\left[a_{g} I_{0}-\left(a_{g}+u_{g}\right) Z-a_{g} C-a_{g} G\right]+m|\sin t| C Z .
\end{gathered}
$$

We consider some numerical experiments which show that not only the periodic orbit persists but also sort of period bifurcation phenomena occur.

For simplicity we fix all the parameters but $I_{0}$ as follows:

$$
u_{g}=u_{c}=m=e=a_{g}=1, \quad a_{c}=3 .
$$




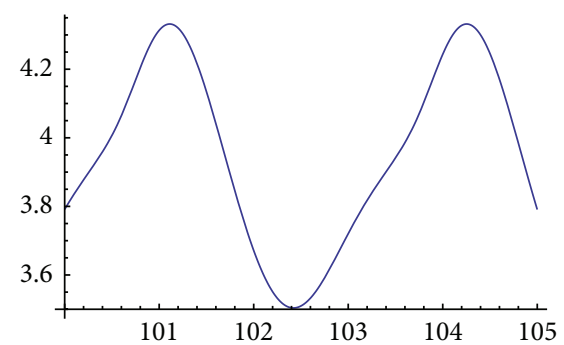

(a)

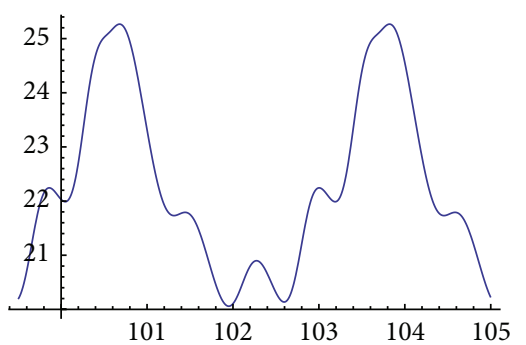

(b)

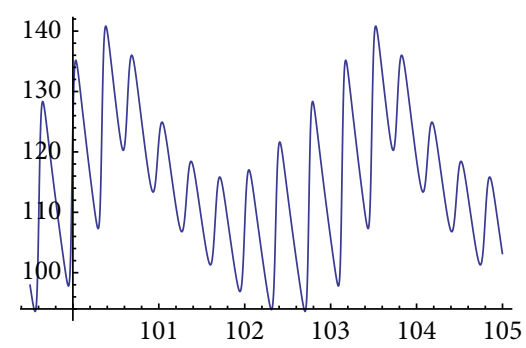

(c)

FIgURE 7: The period of the function $Z$ for $I_{0}=7,35,177$.

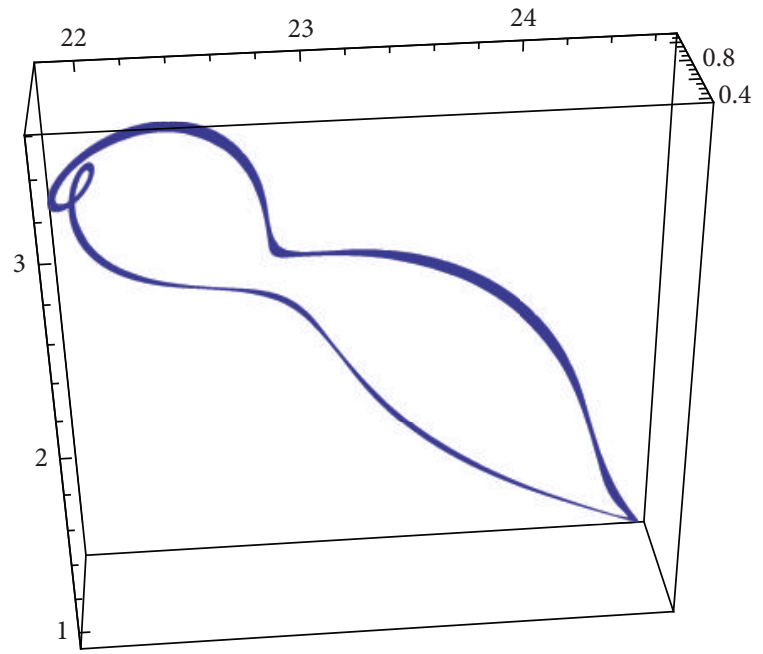

Figure 8: The attractor for $I_{0}=30$.

In Figure 4 we represent the solution for $I_{0}=7,14,28$ while in Figure 5 for $I_{0}=35,82,177$. We note that as $I_{0}$ increases, the period of the solution increases, and in Figure 6 we represent the solutions $Z, C, G$ as function of time for $I_{0}=$ 177 showing different frequencies of oscillations. It would be interesting to analyze this phenomenon in details together with the possibility to have chaotic motion. In Figure 7 we compare the period and oscillations of the function $Z(t)$ for $I_{0}=7,35,177$.

\section{A Generalized Model}

In this section we consider a slighter modification of the model, both in the autonomous and nonautonomous cases. If we sum the second and the third equations of the system, we get

$$
\dot{C}+\dot{G}=\left(a_{c} C+a_{G} G\right)\left(I_{0}-Z-C-G\right)-Z\left(u_{c} C+u_{g} G\right) ;
$$

then the indirect effects terms disappear and so indirect effects do not change the total amount of the preys $C+G$ but only the proportion between them. A simple generalization of the model consists in considering different values of the

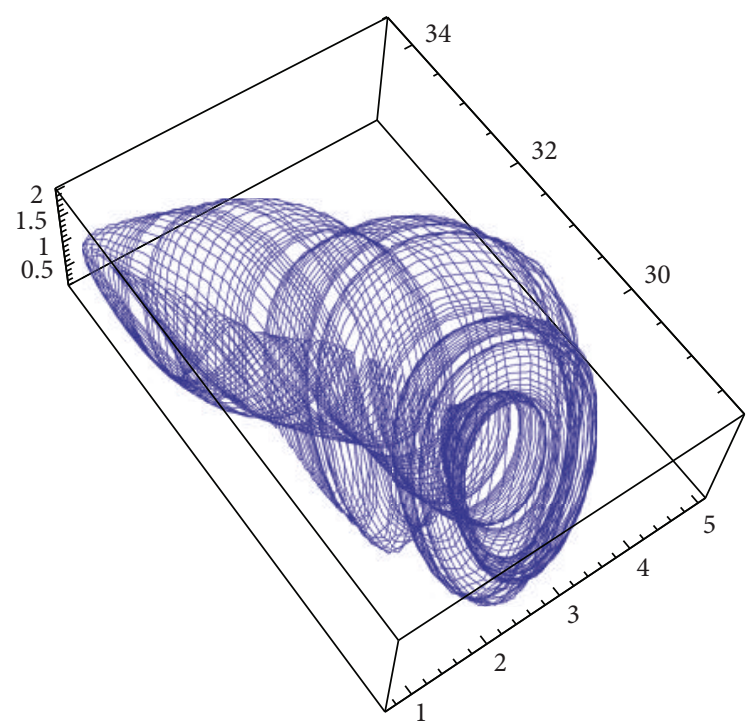

FIgURE 9: The attractor for $I_{0}=40$.

parameter $m$, that is, $m_{1}$ and $m_{2}$, respectively, in the second and the third equations of the system

$$
\begin{gathered}
Z^{\prime}=Z\left(-e+u_{c} C+u_{g} G\right), \\
C^{\prime}=C\left[a_{c} I_{0}-\left(a_{c}+u_{c}\right) Z-a_{c} C-a_{c} G\right]-m_{1} C Z, \\
G^{\prime}=G\left[a_{g} I_{0}-\left(a_{g}+u_{g}\right) Z-a_{g} C-a_{g} G\right]+m_{2} C Z .
\end{gathered}
$$

The dynamical behavior of the system (48) is very similar to the case $m_{1}=m_{2}$ (see [14] for details). In particular it has the same boundary fixed points with the same stability character. Then we can state the following without giving the details of computation.

Theorem 3. The system (48) admits coexistence for any positive solution if the following two conditions are satisfied:

$$
\begin{gathered}
I_{0}>\left\{\frac{e}{u_{c}}, \frac{e}{u_{g}}\right\}, \\
M_{1}=a_{c} u_{g}-a_{g} u_{c}-a_{g} m_{1}>0 .
\end{gathered}
$$

We note that the previous conditions do not depend on the parameter $m_{2}$. The differences between the two models 


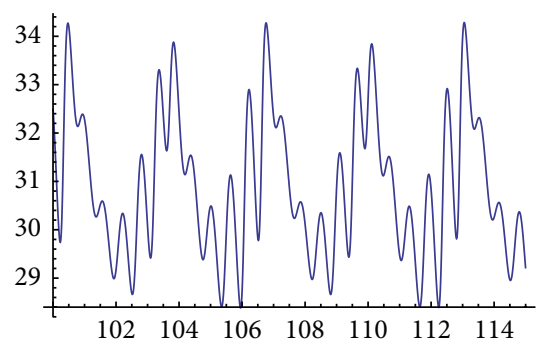

(a)

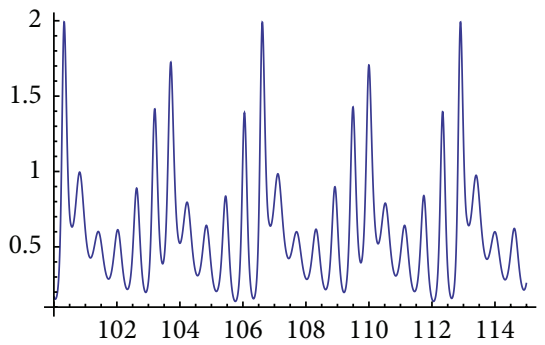

(b)

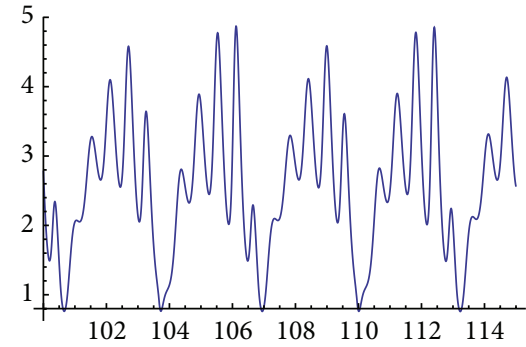

(c)

FIgURE 10: The solutions $Z, C, G$ as function of time for $I_{0}=40$.

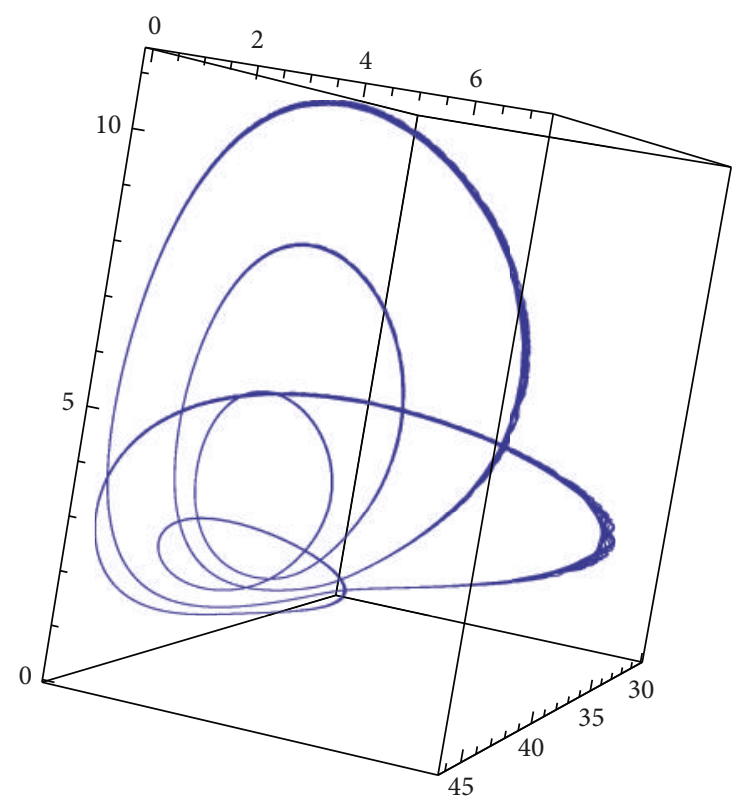

FIGURE 11: The attractor for $I_{0}=50$.

appear in the coordinate of the fixed point $\widetilde{Q}=\left(Z_{\widetilde{Q}}, C_{\widetilde{Q}}, G_{\widetilde{Q}}\right)$ as follows:

$$
\begin{gathered}
G_{\widetilde{Q}}=\left\{\frac{e / u_{c}}{M_{1} / a_{c} m_{2}+u_{g} / u_{c}}\right\}, \\
C_{\widetilde{Q}}=\frac{M_{1}}{a_{c} m_{2}}\left\{\frac{e / u_{c}}{M_{1} / a_{c} m_{2}+u_{g} / u_{c}}\right\}, \\
Z_{\widetilde{Q}}=\frac{a_{c}}{m_{c}^{1}}\left\{I_{0}-\left(\frac{M_{1}}{m_{2} a_{c}}+1\right)\left(\frac{e / u_{c}}{M_{1} / a_{c} m_{2}+u_{g} / u_{c}}\right)\right\},
\end{gathered}
$$

where $m_{c}^{1}=a_{c}+u_{c}+m_{1}$. In particular, as for the original system, if the hypotheses of the previous theorem are satisfied, the interior fixed point $Q$ always exists.

The parameter $m_{2}$ plays a role only in the stability of the point $\widetilde{Q}$ and as a consequence in the structure of the attractor. By the same method of Section 2 it is possible to derive a condition similar to (18) for Hopf bifurcation.
Proposition 4. Suppose that (49) hold. Then the matrix $A(\widetilde{Q})$ has one negative and two purely imaginary eigenvalues if and only if $I_{0}=\widetilde{I}_{0}^{*}$ and

$$
\widetilde{F}-e a_{c}<0,
$$

where

$$
\begin{gathered}
\widetilde{F}=\frac{M_{1}}{m_{2}}\left(\frac{e / u_{c}}{M_{1} / a_{c} m_{2}+u_{g} / u_{c}}\right) \\
\times\left\{u_{c}+\frac{M_{1}}{m_{c}^{1}}+\frac{u_{g} a_{g} m_{2}}{M_{1}}+\frac{m_{2} a_{c}}{m_{c}^{1}}\right\}, \\
\widetilde{D}=G_{\widetilde{Q}}, \\
\widetilde{A}=\left(\frac{M_{1}}{a_{c} m_{2}}+1\right), \\
\widetilde{I}_{0}^{*}=\widetilde{A} \widetilde{D}-\widetilde{D} \frac{\widetilde{F}\left(m_{c}^{1} a_{g} / M_{1}+m_{c}^{1} / m_{2}\right)}{\widetilde{F}-e a_{c}} .
\end{gathered}
$$

We note that inequality (51) depends on $m_{1}$ and $m_{2}$.

From the previous discussion we remark that the behavior of the system (48) is qualitative, the same of that of the system (2).

Numerical simulations suggest that the nonautonomous version of system (48) presents peculiar features. We put $m_{1}|\sin t|$ and $m_{2}|\sin t|$, respectively, in the second and third equations of the system. If $m_{1}>m_{2}$, the numerical experiments showed a behavior of the system similar to that described in the previous section (i.e., the case $m_{1}=m_{2}$ ). If $m_{2}>m_{1}$, we have that

$$
\begin{aligned}
\dot{C}+\dot{G}= & \left(a_{c} C+a_{G} G\right)\left(I_{0}-Z-C-G\right) \\
& -Z\left(u_{c} C+u_{g} G\right)+\left(m_{2}-m_{1}\right)|\sin t|,
\end{aligned}
$$

then, since the last term is nonnegative, we expect a more complicated behavior of the solutions. 


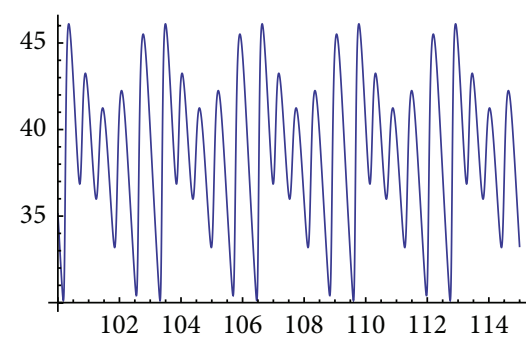

(a)

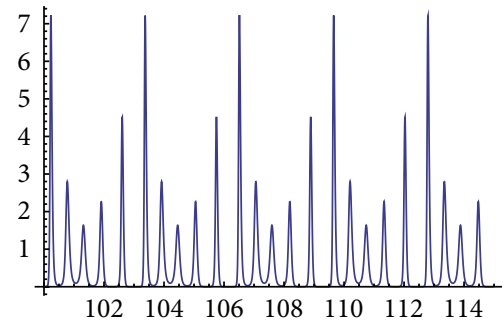

(b)

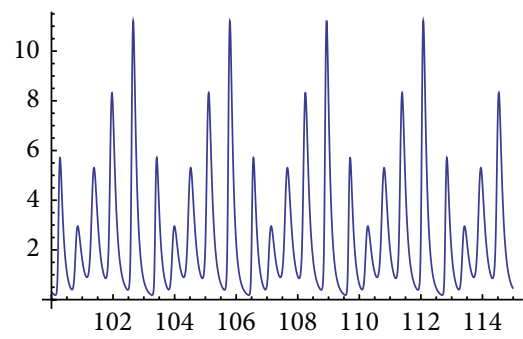

(c)

Figure 12: The solutions $Z, C, G$ as function of time for $I_{0}=50$.

We present some simulations to described the dynamics of this case. We consider the following values of the parameters:

$$
\begin{gathered}
a_{c}=5, \quad a_{g}=\frac{1}{10}, \quad m_{1}=\frac{4}{5}, \quad m_{2}=\frac{3}{2} \\
u_{c}=\frac{8}{10}, \quad u_{g}=\frac{2}{10}, \quad e=1, \\
Z(0)=C(0)=G(0)=\frac{2}{3} .
\end{gathered}
$$

For $I_{0} \geq 1.3$ the attractor is a periodic orbit, and in Figure 8 we represent the case $I_{0}=30$. The most interesting case is when $I_{0}=40$ where a new structure appears; see Figures 9 and 10.

For higher value of $I_{0}$ we obtain an attractor similar to what we have found in the previous section but with a more complicated geometry. In Figures 11 and 12 we represent, respectively, the attractor and the function $Z, C, G$ for $I_{0}=50$.

The numerical experiments suggest that indirect effects encourage an oscillatory behavior of the system and, as a consequence, they are useful to describe real cases of coexistence (see [20]). It could be interesting to investigate in details the nonautonomous case, in particular the phenomenon of period doubling and the existence of chaotic motion (see [2022] for examples for the classical case).

\section{Acknowledgment}

This work was partially supported by the research group EMAP (Grupo de investigaciòn en matemàatica y estadistica aplicada) of Pontificia Universidad Javeriana of Cali, Colombia.

\section{References}

[1] B. Bolker, M. Holyoak, V. Křivan, L. Rowe, and O. Schmitz, "Connecting theoretical and empirical studies of trait-mediated interactions," Ecology, vol. 84, no. 5, pp. 1101-1114, 2003.

[2] D. Cariveau, R. E. Irwin, A. K. Brody, L. S. Garcia-Mayeya, and A. Von Der Ohe, "Direct and indirect effects of pollinators and seed predators to selection on plant and floral traits," Oikos, vol. 104, no. 1, pp. 15-26, 2004.

[3] B. A. Menge, "Indirect effects in marine rocky intertidal interaction webs: patterns and importance," Ecological Monographs, vol. 65 , no. 1, pp. 21-74, 1995.
[4] W. E. Snyder and A. R. Ives, "Generalist predators disrupt biological control by a specialist parasitoid," Ecology, vol. 82, no. 3, pp. 705-716, 2001.

[5] J. T. Wootton, "Indirect effects, prey susceptibility, and habitat selection: impacts of birds on limpets and algae," Ecology, vol. 73, no. 3, pp. 981-991, 1992.

[6] M. R. Walsh and D.N. Reznick, "Interactions between the direct and indirect effects of predators determine life history evolution in a killifish," Proceedings of the National Academy of Sciences of the United States of America, vol. 105, no. 2, pp. 594-599.

[7] D. O. Hessen, T. Andersen, P. Brettum, and B. A. Faafeng, "Phytoplankton contribution to sestonic mass and elemental ratios in lakes: implications for zooplankton nutrition," Limnology and Oceanography, vol. 48, no. 3, 2003.

[8] J. Estes, K. Crooks, and R. Holt, Ecological Role of Predators, vol. 4 of Enciclopedia of Biodiversity, Academic Press, 2001.

[9] V. Lundgren and E. Granéli, "Grazer-induced defense in Phaeocystis globosa (Prymnesiophyceae): influence of different nutrient conditions," Limnology and Oceanography, vol. 55, no. $5,2010$.

[10] M. F. Carusela, F. R. Momo, and L. Romanelli, "Competition, predation and coexistence in a three trophic system," Ecological Modelling, vol. 220, no. 19, pp. 2349-2352, 2009.

[11] Indirect effects affects ecosystem dynamics, 2011, http://www.ictp-saifr.org/.

[12] R. Margalef, "Life forms of Phytoplanktos as survival alternative in an unstable environment," Oceanologica Acta, vol. 134, 1978.

[13] O. Sarnelle, "Daphnia as keystone predators: effects on phytoplankton diversity and grazing resistance," Journal of Plankton Research, vol. 27, no. 12, pp. 1229-1238, 2005.

[14] R. Colucci, "Coexistence in a one-predator, two-prey system with indirect effects," Journal of Applied Mathematics, vol. 2013, Article ID 625391, 13 pages, 2013.

[15] H. L. Smith and H. R. Thieme, Dynamical Systems and Population Persistence, American Mathematical Society, 2011.

[16] G. Butler, H. I. Freedman, and P. Waltman, "Uniformly persistent systems," Proceedings of the American Mathematical Society, vol. 96, no. 3, pp. 425-430, 1986.

[17] S. M. Shahruz and D. A. Kalkin, "Limit cycle behavior in threeor higher-dimensional non-linear systems: the Lotka-Volterra example," Journal of Sound and Vibration, vol. 246, no. 2, pp. 379-386, 2001.

[18] P. Van Den Driessche and M. L. Zeeman, "Three-dimensional competitive Lotka-Volterra systems with no periodic orbits," SIAM Journal on Applied Mathematics, vol. 58, no. 1, pp. 227234, 1998. 
[19] Y. A. Kuznetsov, Elements of Applied Bifurcation Theory, Springer, 1998.

[20] J. Hulsman and F. J. Weissing, "Biodiversity of plankton by species oscillations and chaos," Nature, vol. 402, pp. 407-410, 1999.

[21] J.-M. Ginoux, B. Rossetto, and J.-L. Jamet, "Chaos in a threedimensional Volterra-Gause model of predator-prey type," International Journal of Bifurcation and Chaos in Applied Sciences and Engineering, vol. 15, no. 4, pp. 1689-1708, 2005.

[22] A. Klebanoff and A. Hastings, "Chaos in one-predator, two-prey models: general results from bifurcation theory," Mathematical Biosciences, vol. 122, no. 2, pp. 221-233, 1994. 


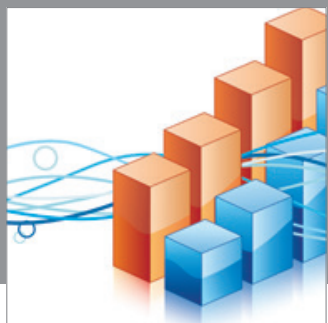

Advances in

Operations Research

mansans

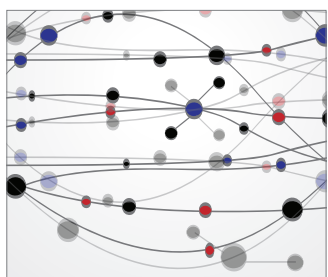

The Scientific World Journal
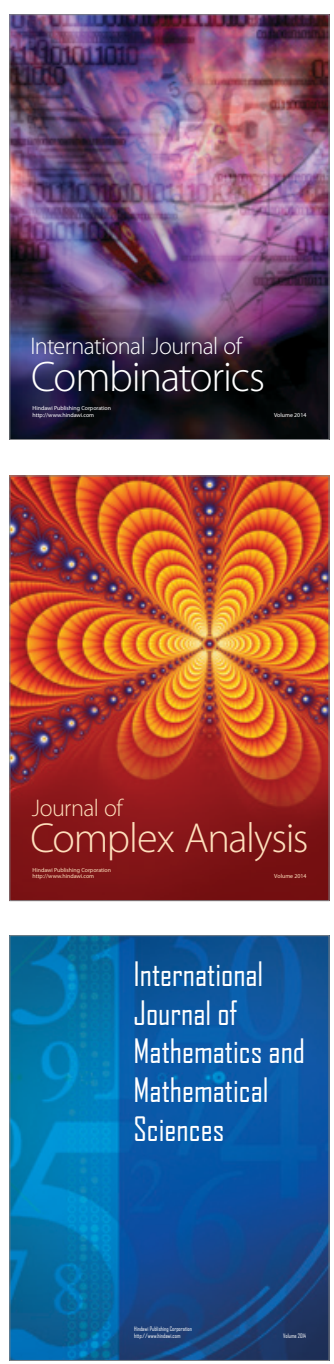
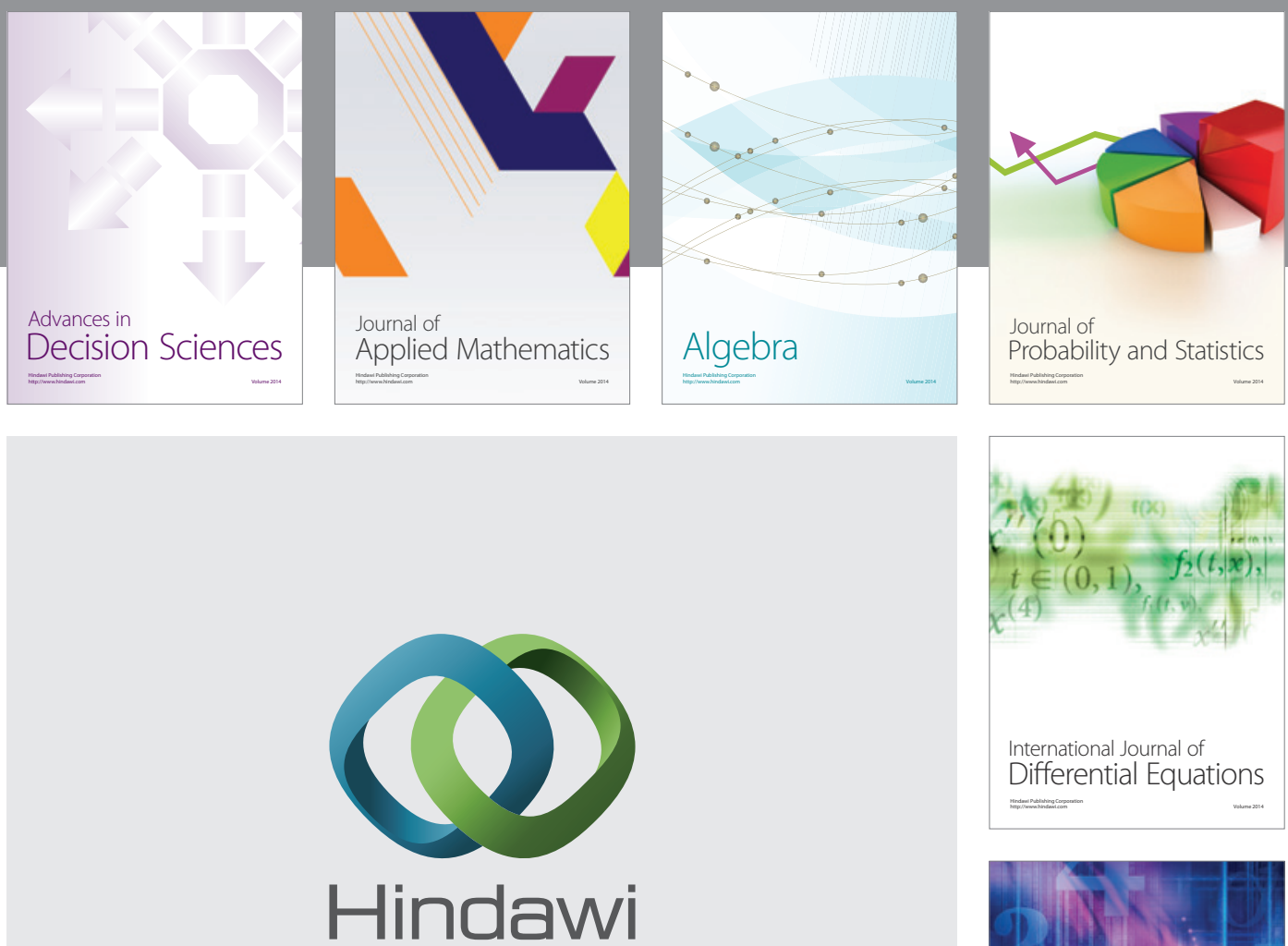

Submit your manuscripts at http://www.hindawi.com
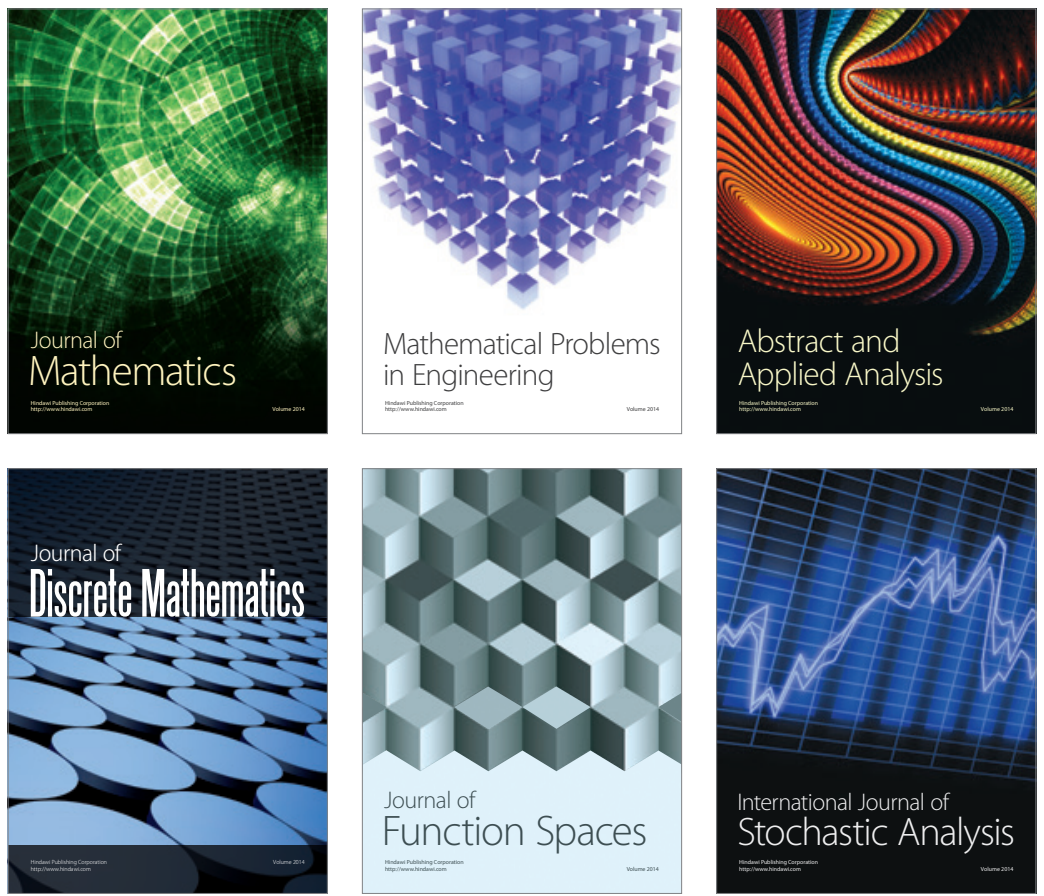

Journal of

Function Spaces

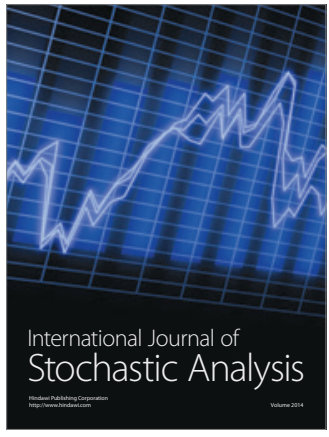

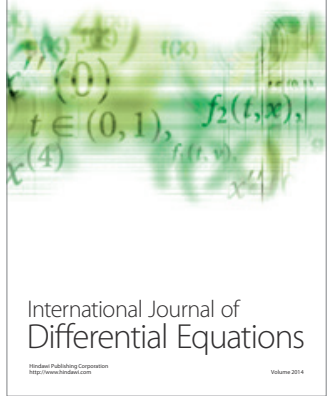
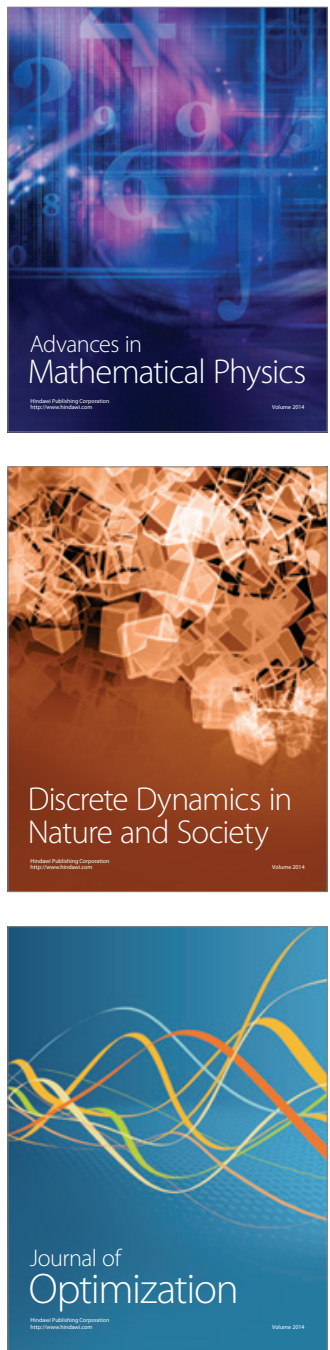